\title{
WIDEBAND ANTENNA FOR HPM MEASUREMENTS
}

\author{
Ivan Kurkan ${ }^{1,2,}$, Nicolay Bykov ${ }^{1}$, Nataliya Kurkan $^{2}$, Alexandr Ryumkin ${ }^{2}$ \\ ${ }^{1}$ Institute of High Current Electronics, 634055, Tomsk, Russia \\ ${ }^{2}$ National Research Tomsk Polytechnic University, 634050, Tomsk, Russia
}

\begin{abstract}
The measurements of microwave pulses of gigawatt power level have a lot of constraints. A receiving antenna is a starting and core point of the measurement system. Waveguide based and dipole antennas have a limited wide bands, while the use of commercially available wideband antennas is restricted by their maximum peak power acceptances. The design of the wide band antenna with the small effective area was proposed. The characteristics of prototype were obtained in numerical simulations with ANSYS HFSS software and by calibration tests in the frequency band of 1-13 GHz. It has the effective area about the $1 \mathrm{~mm} 2$ in $\mathrm{X}$-band and square-law dependence on the wavelength in a wide band. The cross polarization rate is more than $60 \mathrm{~dB}$ at the centre position and not less than $30 \mathrm{~dB}$ within the range of $\pm 5^{0}$ in azimuth and elevation angle. The wide beam radiation pattern forces a user to discriminate reflected signals. This antenna could greatly simplify the measurement system, replacing a set of narrow band antennas that connected to several recording channels.
\end{abstract}

\section{Introduction}

The progress in pulsed power technologies resulted in development of high power microwave (HPM) sources for the last decades. These HPM generators from decimetres to millimetres frequency bands reach the output peak power of $10^{8} \ldots 10^{9} \mathrm{~W}$ with pulse duration of $1 \ldots 100 \mathrm{~ns}[1,2]$. The challenges of such pulses measurement are naturally caused by their high power level, short pulse duration and relatively wide band comparing to continuously operating sources. There are other negative factors featured by feeding sources: the high level of EM noise and bremsstrahlung radiation caused by MeV electron beam. So, a facility requires the presence of radiation shielding and screen room for operators and measurement instruments. It usually makes some constraints on the room available for placement of receiving antenna and requires distant transmission lines to remote screen room. The most of HPM sources operates in the single shot mode or at rather low repetition rates. The measurement of output microwave radiation includes power amplitude, pulse shape, pulse energy, radiation spectrum and pattern. The latter one is directly connected to the operating mode of microwave generator and the internal configuration of electromagnetic fields.

* Corresponding author: ikk@1fe.hcei.tsc.ru 
A receiving antenna is a starting and core point of microwave measurement system. It should be capable to operate at high power density level $\left(\sim 100 \mathrm{~kW} / \mathrm{cm}^{2}\right)$. So, it should be of the low effective area to prevent a breakdown in the transmitting lines, that limits one to use commercially available wide band antennas. It is preferred to be linearly polarized to detect radiation polarization. Horns and waveguide based antennas are widely used as receiving ones at X-band and higher frequencies (Figure 1,a) [2]. The coaxial based dipole antennas are preferable at $\mathrm{L}, \mathrm{S}$ and $\mathrm{C}$-bands as a sizes of waveguides rise with a wavelength (Figure 1,b). Both types of these receiving antennas has the limited operating band. It is restricted by the cut-off frequency and appearing of other than fundamental mode for waveguide. The construction of dipole requires the $\lambda / 4$ - cuts for symmetric operation.

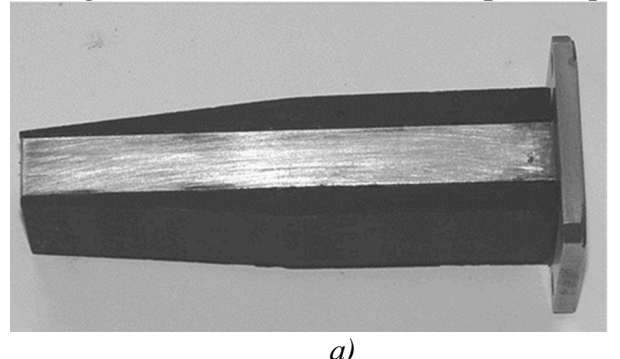

a)

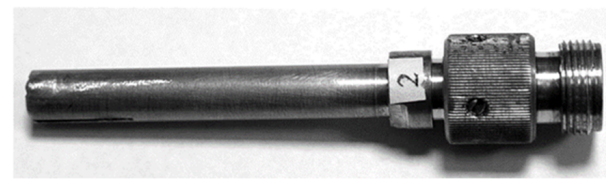

b)

Figure 1. The receiving antenna based on $23 \times 10 \mathrm{~mm}$ waveguide $-a$; the short symmetric dipole with N-type connector $-b$.

During a testing and tuning a HPM generator, experimenters commonly deal with some specific modes of generation those have few concurrent frequencies or valuable harmonics in the output spectrum. These frequencies and particularly harmonics might be out of working band of receiving antenna. So, the usage of a wide band antenna is quite reasonable in this case.

We propose the antenna with the effective area of square millimetres. The main idea was to greatly reduce the receiving element size $L$ comparing to the wavelength $\lambda$, so the effective area would have smooth dependence on the frequency, rising as its square root. It stays correct until $L<\lambda / 4$. We took a coaxial cable as the base and shortcut the central electrode to the shielding with the minimal gap as it is presented in Figure 2. There is the semi-rigid cable FlexiForm 402 by Habia here, but other kinds of $18 \mathrm{GHz}$ semi-rigid or rigid (with the full copper jacket) were also tested and approved.

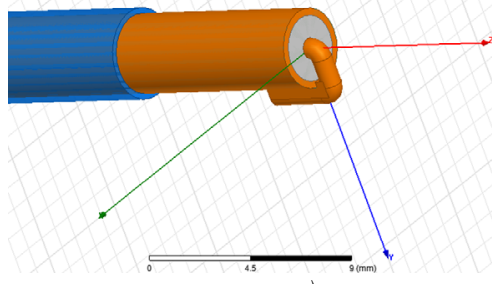

a)

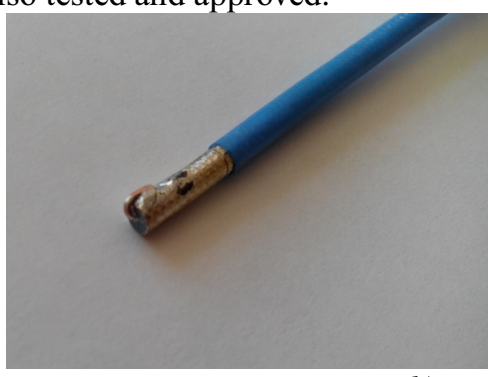

b)

Figure 2. The model of receiving element of antenna $-a$; the receiving element based on semi-rigid cable FlexiForm 402 by Habia $-b$.

We use the pyramidal microwave absorber to cover a cable and connector to prevent surface currents which result in standing waves between the receiving end and the connector. Adsorbing plates on the waveguide based antenna do the same [2]. 
In the paper, the results of numerical simulation and measured characteristics of real antenna we proposed are presented.

\section{Numerical simulation results}

Numerical simulations of this antenna were performed with ANSYS HFSS [3] software in 3D. The antenna was placed in coordinate system as shown in the Figure 2, a, so the working polarization was the $\mathrm{Y}$ one. The task was to prove the selective polarization properties of the antenna and to check its sensitivity to the positioning error within small angles about zero.

The core setup was calculated at $10 \mathrm{GHz}$ by the iterative solver to satisfy S- parameters error of less than $1 \%$, then "discrete sweep" with the same mesh was made to calculate fields at lower frequencies up to $1 \mathrm{GHz}$ with the step of $0.5 \mathrm{GHz}$. The far field radiation patterns were plotted and analysed at calculated frequency points. Figure 3 shows radiation patterns of the antenna at several frequencies as well as pattern at $4 \mathrm{GHz}$ for antenna without the absorbing pyramid to demonstrate its role.

a)

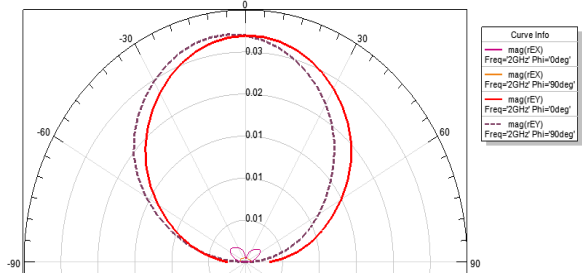

b)

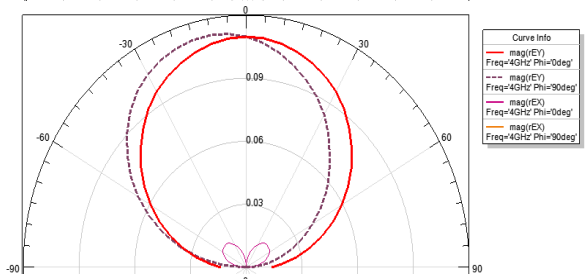

c)

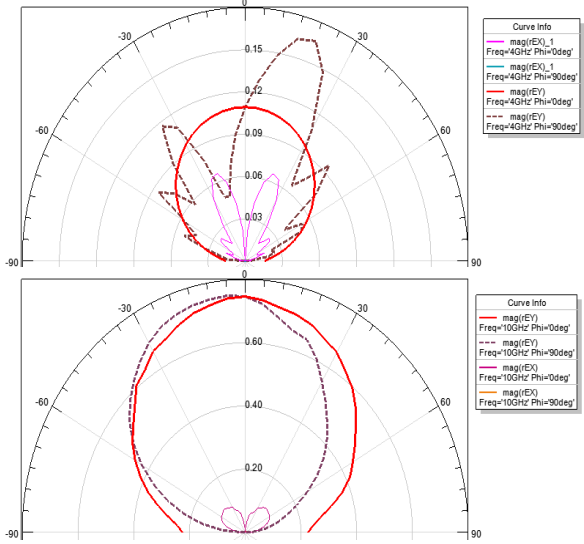

Figure 3. Calculated radiation patterns of the antenna. $a-2 \mathrm{GHz}$ with absorber; $b-4 \mathrm{GHz}$ with absorber; $c-4 \mathrm{GHz}$ w/o absorber; $d-10 \mathrm{GHz}$ with absorber. Legend: bold solid(red) line - rEy component in azimuth plane, bold dashed(brown) line - rEy component in elevation plane, thin solid(magenta) line - rEx component in azimuth plane.

As we are interested in characteristics within small angles about the main direction, the use of Cartesian system for the field description is feasible. The pattern for working Ey 
field polarization is symmetric in the azimuth plane and slightly raised in the elevation plane. The sensitivity within small angles about the working position is stable in the band. The cross polarization rate is more than $60 \mathrm{~dB}$ at the centre position and not worse than 30 $\mathrm{dB}$ within the conus $\pm 5^{0}$. As this antenna has the wide beam radiation pattern, a user should supress reflected signals, using microwave absorbers at the test site.

Figure 3, $c$ represents the radiation pattern for antenna without absorbing cover at frequency of $4 \mathrm{GHz}$. Patterns for other frequencies differ by the number of side lobes. Although the working polarization pattern in azimuthal plane is the same as for absorber covered antenna, patterns in elevation plane and for cross polarized field in azimuth are changed. This antenna became very sensitive to the error in inclination angle positioning and its cross polarization selectivity is much worse, comparing to the absorber covered antenna.

\section{Experiment}

Several antennas based on different type of $18 \mathrm{GHz}$ approved cables were designed, produced and calibrated. All antennas but receiving ends were covered by carbon foam pyramids that were used in microwave anechoic chamber. The main characteristics used by HPM experimenters is the effective area of antenna, which allows one to calculate a power density. The calibration procedure was similar to the one described in the paper [2]. The network analyser Agilent 8719ET was used in the measurement. The difference was in replacing narrow band horn antennas by two ETS-Lindgren's Model 3117 double-ridged waveguide horn antennas with the similar characteristics within the band 1-18 GHz [4]. All standard measures recommended by Agilent to decrease a measurement error were involved: slow frequency scan, 32 passes averaging.

The antennas produced with different types of cables in fact are very close in dimensions, which are predefined by cables frequency band. So, their effective areas are about the same. The effective area dependence on frequency for based on semi-rigid cable FlexiForm 402 by Habia represented at the Figure 4.

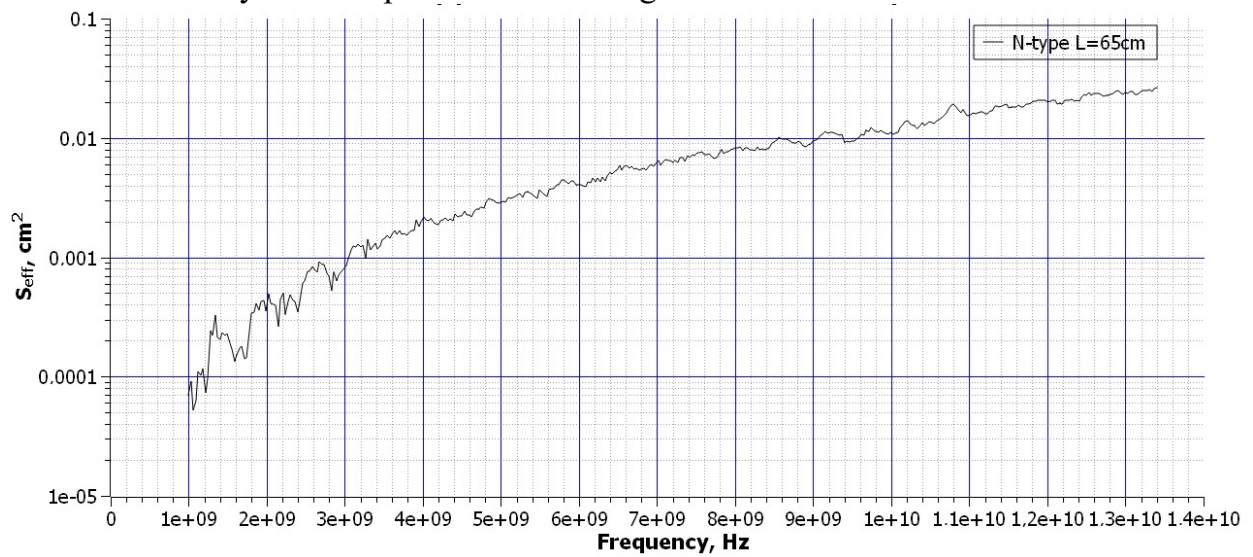

Figure 4. The effective area dependence on frequency for based on semi-rigid cable FlexiForm 402 by Habia.

It confirms the square-law for the effective area dependence on the wavelength. The antenna effective area in X-band is about $1 \mathrm{~mm}^{2}$.

The antenna of this type was used in the experiments with the vircator based on plasmafilled diode, allowing us to monitor its emission in wide band [5] as well as in the 
experiments with the oversized Cherenkov type generator in X-band. The external view of the antenna at the test site is presented in Figure 5.

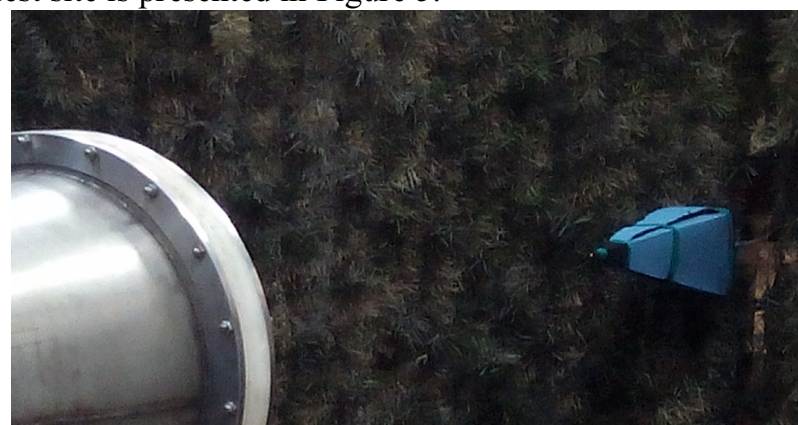

Figure 5. The antenna at the test site.

\section{Conclusion}

The proposed antenna properties were confirmed in numerical simulations and calibration tests. It has the effective area about the $1 \mathrm{~mm}^{2}$ in X-band and square-low dependence on the wavelength in a wide band. The cross polarization rate is more than $60 \mathrm{~dB}$ at the centre position and not less than $30 \mathrm{~dB}$ within the range of $\pm 5^{\circ}$ in azimuth and elevation angle. It has rather wide main lobe of the directivity pattern, so the special measures should be taken to discriminate the reflected signals. It became the advantage, while working in microwave anechoic chamber, as some positioning errors do not result in to a valuable measurement error. This antenna could simplify the measurement of the wide band HPM radiation characteristics, using the single antenna instead of a set of narrow band ones. The effective area rise with the frequency could be partially compensated by a long transmission line attenuation, which also rises with the frequency also, increasing a dynamic resolution of the measurement system as a whole.

\section{Acknowledgements}

We thank our colleague Alexey I. Klimov for assistance with the calibration methodology and plentiful discussions of results. We are also grateful to our colleagues from Tomsk State University of Control System and Radioelectronics, especially to Alexey V. Fateev, for sharing the ETS-Lindgren's antennas. This work was partially supported by RFBR under Grant \#14-08-01123a.

\section{References}

[1] A.I. Klimov, Russ. Phys. J. 39, 1241 (1996) doi:10.1007/BF02436167

[2] A.I. Klimov, O.B. Kovalchuk, V.V. Rostov, A.N. Sinyakov, IEEE Trans. Plasma Sci. 36, 661 (2008) doi: 10.1109/TPS.2008.917300

[3] High Frequency Structure Simulator (HFSS) (Ansys Corporation, Pittsburgh, PA, 15317), May 2016. URL: http://www.ansys.com/Products/Electronics/ANSYSHFSS

[4] EMC antennas. Double-ridged waveguide horn. Model 3117. (ETS-Lindgren Inc., 1301 Arrow Point Drive, Cedar Park, Texas 78613, USA) URL: http://www.etslindgren.com/pdf/3117.pdf

[5] A.A. Zherlitsyn, I.K. Kurkan, Russ. Phys. J. (to be published) 